\title{
AN AXIOMATIZATION FOR TWO POWER INDICES FOR (3,2)-SIMPLE GAMES
}

\author{
JOSEP FREIXAS
}

May 15, 2019

\begin{abstract}
The aim of this work is to give a characterization of the ShapleyShubik power index for (3,2)-simple games. We generalize to the set of $(3,2)$-simple games the classical axioms for power indices on simple games: transfer, anonymity, null player property and efficiency. However, these four axioms are not enough to uniquely characterize the Shapley-Shubik index for (3,2)-simple games. Thus, we introduce a new axiom to prove the uniqueness of the extension of the Shapley-Shubik power index in this context. Moreover, we provide an analogous axiom to characterize also the Banzhaf index for (3,2)-simple games.
\end{abstract}

Keywords: Games with abstention; Power indices; Axioms; Voting.

\section{Introduction}

In classical cooperative theory, simple games are used to model voting situation. However in a simple game players can vote only either yes or no, while in many real-life voting procedure voters are allowed to have other opinions. For instance, it is possible to take into account the possibility of abstention. A theoretical model for game with abstention was introduced in [Felsenthal and Machover [1997]] and extended to voting rules with several levels of approval in input and different levels of output, called $(j, k)$-games in [Freixas and Zwicker [2003]]. A voting procedure with abstention can be seen as a (3,2)-simple games: voters can choose among three different options (namely, voting yes, abstaining and voting no) and the outcome is 0 or 1 .

The Shapley-Shubik index, presented in [Shapley and Shubik [1954]], and the Banzhaf index, defined independently in [Penrose [1946]] and in [Banzhaf [1964]], are the most well-known and widely accepted ways to measure the power of players in simple games. Two indices analogous to these ones have been defined for (3,2)-simple games to measure the power of players also in this specific voting situation. In particular the Shapley-Shubik power index for (3,2)-simple games was defined by [Felsenthal and Machover [1997]] and extended to $(j, k)$ games 
in [Freixas [2005b]]; while the Banzhaf index for (3,2)-simple games is discussed in [Felsenthal and Machover [1998]] and for $(j, k)$ games in [Freixas [2005a]].

A classical axiomatization of these two power indices for simple games has been provided in [Dubey [1975]] and in [Dubey and Shapley [1979]]. The axioms used to characterize the indices are anonymity, transfer, null player, efficiency for the Shapley-Shubik index, and Banzhaf total power for the Banzhaf index. The aim of this work is to provide a characterization for the Shapley-Shubik and the Banzhaf indices for (3,2)-simple games, extending the classical axioms from simple games to $(3,2)$-simple games. However, as we shall discuss, these classical axioms generalized in the context of $(3,2)$-simple games are not enough to uniquely characterize the indices on the space of $(3,2)$-simple games. It is necessary to add another property to describe the behavior of the power index on unanimity games.

The Shapley-Shubik and the Banzhaf indices can be defined on unanimity games and then extended to the family of simple games using the transfer axiom, actually this property holds for any semivalue, as discussed in [Carreras et al. [2003]] and in [Bernardi and Lucchetti [2015]]. The behavior on unanimity games is crucial in order to uniquely characterize an index. The axioms we propose compare the power of a player in a unanimity game when votes "yes" in a minimal winning tripartition and when he abstains in the same situation, i.e. all other players do not change their vote. We give different conditions for the differences of power in the two situations and use these conditions to deduce the Shapley-Shubik and the Banzhaf index for $(3,2)$-simple games, respectively.

The paper is organized as follows. In next section we introduce some preliminaries and definitions. In section 3 we discuss axioms. In section 4 we prove the characterization of the Shapley-Shubik index for (3,2)-simple games and the independence of the axioms used. The analogous results for the Banzhaf index for $(3,2)$-simple games are discussed in section 5. Section 6 concludes the work.

\section{Preliminaries and Definitions}

A game with abstention is a model of a voting situation in which players have three different possibilities: voting "yes", voting "no", and abstaining. This is a generalization of the standard model of simple games in which players can only vote in support of or against the status quo.

Given a finite set of players $N$, in simple games the set $2^{N}$ represents the set of all coalitions. Actually any coalition $T \in 2^{N}$ can be seen as a bipartition $\left(T_{1}, T_{2}\right)$ in which $T_{1}=T$ and $T_{2}=N \backslash T$. We can view a coalition $T$ as the set of players supporting a decision and the coalition $N \backslash T$ as the set of players against it. Analogously, in the context of (3,2)-simple games we consider the set $3^{N}$ of all tripartitions. By tripartition we denote any element $S=\left(S_{1}, S_{2}, S_{3}\right)$, where $S_{1}, S_{2}, S_{3}$ are mutually disjoint subsets of $N$ such that $S_{1} \cup S_{2} \cup S_{3}=N$, any $S_{i}$ can be empty. An element $S=\left(S_{1}, S_{2}, S_{3}\right) \in 3^{N}$ describes a voting situation in which the players in $S_{i}$ are voting at "level $i$ " of approval. It is supposed that level 1 is the highest, level 2 is the intermediate, and level 3 is 
the lowest. Hereafter, with the idea of modelling a voting situation, we will say that players in $S_{1}$ are voting "yes", players in $S_{2}$ are abstaining, players in $S_{3}$ are voting "no".

A partial order $\subseteq$ on the set $3^{N}$ is defined as follows. If $S, T \in 3^{N}$, then $S \subseteq T$ means $S_{1} \subseteq T_{1}$ and $S_{2} \subseteq T_{1} \cup T_{2}$. We use $S \subset T$ if $S \subseteq T$ and $S \neq T$. In other words, a tripartition $S$ is contained in the tripartition $T$ if players in $T$ are voting as in $S$ or some of them are increasing their level of support. This means that $S$ can be transformed into $T$ by shifting one or more players to higher levels of approval. For instance ${ }^{1}(a, b, c) \subseteq(a b, c, \emptyset)$, the second tripartition is obtained by the first one when player $b$ changes from abstaining to voting "yes" and player $c$ switches from voting "no" to abstaining. The tripartition $(\emptyset, \emptyset, N)$ is the minimum of the order $\subseteq$, while the maximum is the tripartition $(N, \emptyset, \emptyset)$.

A (3,2)-simple game is a pair $(N, v)$ in which $N$ is the set of players (or voters) and $v: 3^{N} \rightarrow\{0,1\}$ is the value function such that:

- it is monotonic, i.e. if $S \subseteq T$ then $v(S) \leq v(T)$;

- $v(\emptyset, \emptyset, N)=0$ and $v(N, \emptyset, \emptyset)=1$.

We denote with $\mathfrak{T}^{N}$ the set of all $(3,2)$-simple games on the finite set $N$.

As for simple games, any game $v \in \mathfrak{T}^{N}$ can be described by the set of winning tripartitions $\mathcal{W}(v)=\left\{S \in 3^{N}: v(S)=1\right\}$ or by the set of minimal winning tripartitions $\mathcal{W}^{m}(v)=\left\{S \in 3^{N}: v(S)=1, v(T)=0, \forall T \subset S\right\}$.

[Unanimity game] For any tripartition $S \neq(\emptyset, \emptyset, N)$, the unanimity game $u_{S}$ is defined as

$$
u_{S}(T)=\left\{\begin{array}{l}
1 \text { if } S \subseteq T \\
0 \text { otherwise }
\end{array}\right.
$$

Given a tripartition $S=\left(S_{1}, S_{2}, S_{3}\right)$ and a player $a \notin S_{3}$ we denote with $S_{\downarrow a}$ the tripartition in which player $a$ decreases his or her support of one level

$$
S_{\downarrow a}=\left\{\begin{array}{l}
\left(S_{1} \backslash\{a\}, S_{2} \cup\{a\}, S_{3}\right) \text { if } a \in S_{1} \\
\left(S_{1}, S_{2} \backslash\{a\}, S_{3} \cup\{a\}\right) \text { if } a \in S_{2} .
\end{array}\right.
$$

Of course, there is also the possibility that player $a \in S_{1}$ switches from supporting a decision to vote against it

$$
S_{\downarrow \downarrow a}=\left(S_{1} \backslash\{a\}, S_{2}, S_{3} \cup\{a\}\right) .
$$

In an analogous way, given $S$ and a player $a \notin S_{1}$, we define the tripartition in which $a$ increases the support

$$
S_{\uparrow a}=\left\{\begin{array}{l}
\left(S_{1} \cup\{a\}, S_{2} \backslash\{a\}, S_{3}\right) \text { if } a \in S_{3} \\
\left(S_{1}, S_{2} \cup\{a\}, S_{3} \backslash\{a\}\right) \text { if } a \in S_{3}
\end{array}\right.
$$

\footnotetext{
${ }^{1}$ To simplify the notation we omit the braces to denote the sets in a tripartition, for instance the informal notation $(a, b, c)$ stands for $(\{a\},\{b\},\{c\})$.
} 
and if $a \in S_{3}$

$$
S_{\uparrow \uparrow a}=\left(S_{1} \cup\{a\}, S_{2}, S_{3} \backslash\{a\}\right) .
$$

It is possible to characterize some players according to their role in the game. In the following definition we describe players that do not have influence at all in a voting situation: either they support or not a proposal, they can not change the outcome of the game. [Null player] A player $a \in N$ is null in the game $v$ if $v(S)=v\left(S_{\downarrow \downarrow a}\right)$ for any tripartition $S$ such that $a \in S_{1}$. Note that $a$ is a null player if and only if it holds $v(S)=v\left(S_{\downarrow a}\right)$ for any tripartition $S$ such that $a \in S_{1} \cup S_{2}$. Note also that the previous definition is equivalent to say that $a$ is a null player if $a \in S_{3}$ for any $S \in \mathcal{W}^{m}(v)$.

In the context of simple games, that describe a voting situation, one of the key point is to evaluate the power of players, in order to establish how their vote influences the outcome of the game. For this reason, in simple games, the interesting class of solution concepts is the one of power indices. In the following we define this family on the set of $(3,2)$-simple games and then focus on the extension of two main power indices.

[Power index] A power index for (3,2)-simple games is a function $\psi: \mathfrak{T}^{N} \rightarrow$ $\mathbb{R}^{n}$, that assigns to every game $v$ a vector in which the $a^{\text {th }}$ component is a measure of the power of player $a$ in the voting system described by $v$.

As for simple games, there are different power indices for (3,2)-simple games to capture different properties. The well-known Banzhaf [Banzhaf [1964]] and Shapley-Shubik [Shapley and Shubik [1954]] power indices have an equivalent in the context of (3,2)-simple games, as defined in [Felsenthal and Machover [1997]] and [Freixas [2005a]].

[Banzhaf index for $(3,2)$-simple games] For any game $v \in \mathfrak{T}^{N}$ and any player $a \in N$, define $\eta_{a}(v)$ as the number of yes-no swings for player $a$, that is

$$
\eta_{a}(v)=\mid\left\{S: a \in S_{1} \text { and } v(S)-v\left(S_{\downarrow \downarrow a}\right)=1\right\} \mid .
$$

The Banzhaf index for (3,2)-simple games is then given by

$$
\beta_{a}(v)=\frac{\eta_{a}(v)}{3^{n-1}} .
$$

The Shapley-Shubik power index for (3,2)-simple games was introduced in [Felsenthal and Machover [1997]] using the idea of roll-calls. Let $\mathbf{Q}_{N}$ be the space of all the permutation of $N$, and let $3^{N}$ be the set of all tripartitions of $N$. The ternary roll-call space $\mathbf{R}_{N}$ is defined as

$$
\mathbf{R}_{N}=\mathbf{Q}_{N} \times 3^{N} .
$$

Each roll-call $\mathcal{R}$ is given by a queue $q R$ and a tripartition $R$, that is $\mathcal{R}=(q R, R)$ where $q R$ represents the order in which players are voting and $R$ represents how each one of them is voting. For instance, $q R(a)=i$ means that $a$ is the $i^{t h}$ to vote and $a \in R_{1}$ means that $a$ is voting "yes". The number of the elements in $\mathbf{R}_{N}$ is $n ! 3^{n}$. 
A player $a$ is said to be pivotal in $\mathcal{R}$ for the game $v$ (and we write $\operatorname{piv}(\mathcal{R}, v)$ ) if after $a$ 's vote the outcome is decided, no matter what the players after $a$ in $q R$ are going to vote.

[Shapley-Shubik index for $(3,2)$-simple games] For any $v \in \mathfrak{T}^{N}$ and any player $a \in N$, the Shapley-Shubik index for (3,2)-simple games is defined as

$$
\phi_{a}(v)=\frac{\left|\left\{\mathcal{R} \in \mathbf{R}_{N}: a=\operatorname{piv}(\mathcal{R}, v)\right\}\right|}{3^{n} n !} .
$$

For the purpose of this paper we introduce some notation related to rollcalls. Consider the set of roll-calls $\mathbf{R}_{N}$; for any player $a \in N$, we define the following subsets which form a partition of $\mathbf{R}_{N}$ :

$$
\begin{aligned}
& \mathcal{R}_{a}^{\text {yes }}=\left\{\mathcal{R}: a \in R_{1}\right\}=\{\text { roll-calls in which player } a \text { votes "yes" }\} \\
& \left.\mathcal{R}_{a}^{a b s}=\left\{\mathcal{R}: a \in R_{2}\right\}=\text { \{roll-calls in which player } a \text { abstains }\right\} \\
& \mathcal{R}_{a}^{n o}=\left\{\mathcal{R}: a \in R_{3}\right\}=\{\text { roll-calls in which player } a \text { votes "no" }\}
\end{aligned}
$$

Thus,

$$
\mathbf{R}_{N}=\mathcal{R}_{a}^{\text {yes }} \cup \mathcal{R}_{a}^{a b s} \cup \mathcal{R}_{a}^{n o}
$$

and $\left|\mathcal{R}_{a}^{\text {yes }}\right|=\left|\mathcal{R}_{a}^{a b s}\right|=\left|\mathcal{R}_{a}^{n o}\right|=n ! 3^{n-1}$

Given a player $a$ and a roll-call $\mathcal{R}=(q R, R) \notin \mathcal{R}_{a}^{n o}$, we define the roll-call $\mathcal{R}_{\downarrow a}$ in which players are in the same order as in $\mathcal{R}$, all players in $N \backslash\{a\}$ vote as in $\mathcal{R}$, while $a$ decreases the support of one level

$$
\mathcal{R}_{\downarrow a}=\left(q R, R_{\downarrow a}\right) .
$$

Note that if $\mathcal{R} \in \mathcal{R}_{a}^{\text {yes }}$, then $\mathcal{R}_{\downarrow a} \in \mathcal{R}_{a}^{a b s}$; if $\mathcal{R} \in \mathcal{R}_{a}^{a b s}$, then $\mathcal{R}_{\downarrow a} \in \mathcal{R}_{a}^{n o}$.

We also define the roll-call in which $a$ decreases the support of two levels, changing the vote from "yes" to "no": if $\mathcal{R} \in \mathcal{R}_{a}^{\text {yes }}$, then $\mathcal{R}_{\downarrow \downarrow a} \in \mathcal{R}_{a}^{\text {no }}$ is

$$
\mathcal{R}_{\downarrow \downarrow a}=\left(q R, R_{\downarrow \downarrow a}\right)
$$

For a roll-call $\mathcal{R} \in \mathcal{R}_{a}^{\text {yes }}$ we analogously define $\mathcal{R}_{\uparrow a}=\left(q R, R_{\uparrow a}\right)$ and if $\mathcal{R} \in \mathcal{R}_{a}^{n o}$ we define $\mathcal{R}_{\uparrow \uparrow a}=\left(q R, R_{\uparrow \uparrow a}\right)$.

Note that, for instance, if $\mathcal{R} \in \mathcal{R}_{a}^{\text {yes }}$ we have: $\left(\mathcal{R}_{\downarrow a}\right)_{\uparrow a}=\mathcal{R}$, which shows that there is a one-to-one correspondence between $\mathcal{R}_{a}^{\text {yes }}$ and $\mathcal{R}_{a}^{a b s}$ with these changes. In addition, from $\left(\mathcal{R}_{\downarrow \downarrow a}\right)_{\uparrow \uparrow a}=\mathcal{R}$, the one-to-one correspondence of the three sets $\mathcal{R}_{a}^{\text {yes }}, \mathcal{R}_{a}^{a b s}, \mathcal{R}_{a}^{\text {no }}$ follows.

We also introduce the following sets, for any player $a \in N$ and any game $v$ :

$$
\begin{aligned}
Y_{a, v} & =\left\{\mathcal{R} \in \mathcal{R}_{a}^{\text {yes }}: a=\operatorname{piv}(\mathcal{R}, v)\right\} \\
A_{a, v} & =\left\{\mathcal{R} \in \mathcal{R}_{a}^{a b s}: a=\operatorname{piv}(\mathcal{R}, v)\right\} \\
N_{a, v} & =\left\{\mathcal{R} \in \mathcal{R}_{a}^{n o}: a=\operatorname{piv}(\mathcal{R}, v)\right\} .
\end{aligned}
$$


and the following subsets of $A_{a, v}$ and $N_{a, v}$ :

$$
\begin{aligned}
A Y_{a, v}=\left\{\mathcal{R} \in A_{a, v}: \mathcal{R}_{\uparrow a} \in Y_{a, v}\right\} & A \bar{Y}_{a, v}=\left\{\mathcal{R} \in A_{a, v}: \mathcal{R}_{\uparrow a} \notin Y_{a, v}\right\} \\
N Y_{a, v}=\left\{\mathcal{R} \in N_{a, v}: \mathcal{R}_{\uparrow \uparrow a} \in Y_{a, v}\right\} & N \bar{Y}_{a, v}=\left\{\mathcal{R} \in N_{a, v}: \mathcal{R}_{\uparrow \uparrow a} \notin Y_{a, v}\right\} .
\end{aligned}
$$

Thanks to the previous notation, the Shapley-Shubik index as defined in (1) can be written as

$$
\phi_{a}(v)=\frac{1}{3^{n} n !}\left[\left|Y_{a, v}\right|+\left|A_{a, v}\right|+\left|N_{a, v}\right|\right]
$$

or as

$$
\phi_{a}(v)=\frac{1}{3^{n} n !}\left[\left|Y_{a, v}\right|+\left|A Y_{a, v}\right|+\left|A \bar{Y}_{a, v}\right|+\left|N Y_{a, v}\right|+\left|N \bar{Y}_{a, v}\right|\right] .
$$

Lastly, given two games $v, w \in \mathfrak{T}^{N}$, we define the following games:

Disjunction: the game $v \vee w$ is defined as $(v \vee w)(S)=\max \{v(S), w(S)\}$

Conjunction: the game $v \wedge w$ is defined as $(v \wedge w)(S)=\min \{v(S), w(S)\}$.

Let us make some remarks about these operations:

$W(v \vee w)=W(v) \cup W(w)$ and $W(v \wedge w)=W(v) \cap W(w) ;$

if $W^{m}(v)=\left\{S_{1}, \ldots, S_{k}\right\}$ then $v=u_{S_{1}} \vee \cdots \vee u_{S_{k}}$;

given two unanimity games $u_{S}$ and $u_{T}$, then their conjunction is still a unanimity game and in particular $u_{S} \wedge u_{T}=u_{Z}$ with $Z_{1}=S_{1} \cup T_{1}, Z_{2}=\left(S_{2} \cup T_{2}\right) \backslash Z_{1}$ and $Z_{3}=N \backslash\left(Z_{1} \cup Z_{2}\right)$.

In Table 1 the Shapley-Shubik and the Banzhaf indices are computed for all the (3,2)-simple games (up to isomorphism) on $N=\{1,2\}$.

\section{Axioms for power indices for (3,2)-simple games}

\subsection{Classical axioms for (3,2)-simple games}

In the following, $\psi: \mathfrak{T}^{N} \rightarrow \mathbb{R}^{n}$ is a power index for (3,2)-simple games.

Anonymity The index $\psi$ satisfies anonymity if for all game $v \in \mathfrak{T}^{N}$, any permutation $\pi$ of $N$ and any $a \in N$

$$
\psi_{a}(v)=\psi_{\pi(a)}(\pi v)
$$

where $(\pi v)(S)=v(\pi(S))$.

Null Player The index $\psi$ satisfies the null player axiom if given a null player $a$ in the game $v$, then

$$
\psi_{a}(v)=0 .
$$

Transfer The index $\psi$ satisfies transfer if for any $v, w \in \mathfrak{T}^{N}$

$$
\psi(v)+\psi(w)=\psi(v \wedge w)+\psi(v \vee w) .
$$




\begin{tabular}{|c|c|c|c|}
\hline & $W^{m}$ & S-S index & Bz index \\
\hline 1 & $(12, \emptyset, \emptyset)$ & $\left(\frac{1}{2}, \frac{1}{2}\right)$ & $\left(\frac{1}{3}, \frac{1}{3}\right)$ \\
2 & $(1,2, \emptyset)$ & $\left(\frac{2}{3}, \frac{1}{3}\right)$ & $\left(\frac{2}{3}, \frac{1}{3}\right)$ \\
3 & $(1,2, \emptyset)$ and $(2,1, \emptyset)$ & $\left(\frac{1}{2}, \frac{1}{2}\right)$ & $\left(\frac{2}{3}, \frac{2}{3}\right)$ \\
4 & $(1,2, \emptyset)$ and $(2, \emptyset, 1)$ & $\left(\frac{1}{6}, \frac{5}{6}\right)$ & $\left(\frac{1}{3}, \frac{1}{3}\right)$ \\
5 & $(1, \emptyset, 2)$ & $(1,0)$ & $(1,0)$ \\
6 & $(1, \emptyset, 2)$ and $(2, \emptyset, 1)$ & $\left(\frac{1}{2}, \frac{1}{2}\right)$ & $\left(\frac{2}{3}, \frac{2}{3}\right)$ \\
7 & $(1, \emptyset, 2)$ and $(\emptyset, 12, \emptyset)$ & $\left(\frac{5}{6}, \frac{1}{6}\right)$ & $\left(1, \frac{2}{3}\right)$ \\
8 & $(1, \emptyset, 2)$ and $\emptyset, 2,1)$ & $\left(\frac{1}{3}, \frac{2}{3}\right)$ & $\left(\frac{1}{3}, \frac{2}{3}\right)$ \\
9 & $(\emptyset, 12, \emptyset)$ & $\left(\frac{1}{2}, \frac{1}{2}\right)$ & $\left(\frac{2}{3}, \frac{2}{3}\right)$ \\
10 & $(\emptyset, 1,2)$ & $(1,0)$ & $(1,0)$ \\
11 & $(\emptyset, 1,2)$ and $(\emptyset, 2,1)$ & $\left(\frac{1}{2}, \frac{1}{2}\right)$ & $\left(\frac{2}{3}, \frac{2}{3}\right)$ \\
12 & $(1, \emptyset, 2)$ and $(2, \emptyset, 1)$ and $(\emptyset, 12, \emptyset)$ & $\left(\frac{1}{2}, \frac{1}{2}\right)$ & $\left(\frac{2}{3}, \frac{2}{3}\right)$ \\
\hline
\end{tabular}

Table 1: Shapley-Shubik and Banzhaf indices for (3,2)-simple games with two players.

Efficiency The index $\psi$ satisfies efficiency if for any $v \in \mathfrak{T}^{N}$

$$
\sum_{a \in N} \psi_{a}(v)=1
$$

Banzhaf Total Power The index $\psi$ satisfies Banzhaf total power if for any $v \in \mathfrak{T}^{N}$

$$
\sum_{a \in N} \psi_{a}(v)=\frac{1}{3^{n-1}} \sum_{a=1}^{n} \sum_{\substack{S \in 3^{N} \\ a \in S_{1}}}\left[v(S)-v\left(S_{\downarrow \downarrow a}\right)\right] .
$$

As for simple games, both Shapley-Shubik and Banzhaf indices for $(3,2)$ simple games satisfy anonymity, null player and transfer. Moreover, the ShapleyShubik index for $(3,2)$-simple games satisfies also efficiency, while the Banzhaf index for (3,2)-simple games satisfies the tautological Banzhaf total power axiom. However, as we will discuss later on, these axioms are not sufficient to characterize the Shapley-Shubik and the Banzhaf indices for (3,2)-simple games.

The Shapley-Shubik index for (3,2)-simple games satisfies the anonymity, the null player, the transfer and the efficiency axioms.

Let us consider the different properties.

Anonymity Let $\pi$ be a permutation of $N$. Given a roll-call $\mathcal{R}=(q R, R)$, define $\pi(\mathcal{R})=(\pi(q R), \pi(R))$. This means that if $\pi(a)=b$ then $b$ votes in $\pi(\mathcal{R})$ in the same position and in the same level of approval of $a$ in $\mathcal{R}$. 
If $a$ is pivotal in the game $v$ for the roll-call $\mathcal{R}$, then $\pi(a)$ is pivotal in the game $\pi v$ for the roll-call $\pi(\mathcal{R})$. Then

$\phi_{a}(v)=\frac{|\{\mathcal{R}: a=\operatorname{piv}(\mathcal{R}, v)\}|}{3^{n} n !}=\frac{|\{\pi(\mathcal{R}): \pi a=\operatorname{piv}(\pi \mathcal{R}, \pi v)\}|}{3^{n} n !}=\phi_{\pi(a)}(\pi v)$.

So the Shapley-Shubik index for $(3,2)$-simple games satisfies the anonymity axiom.

Null player If $a$ is a null player in a game $v$, there is not a roll-call $\mathcal{R}$ such that $a=\operatorname{piv}(\mathcal{R}, v)$. Then $\phi_{a}(v)=0$.

Transfer Let $v$ and $w$ be two (3,2)-simple games, then consider the following sets of roll-calls:

$$
\begin{aligned}
& A=\{\mathcal{R}: a \text { is pivotal in } v \text { and in } w\} \\
& B=\{\mathcal{R}: a \text { is pivotal in } v \text { but not in } w\} \\
& C=\{\mathcal{R}: a \text { is pivotal in } w \text { but not in } v\} .
\end{aligned}
$$

Note that $A$ and $B$ form a partition of the set of roll-calls for which $a$ is pivotal in $v$, while $A$ and $C$ form a partition of the set of roll-calls for which $a$ is pivotal in $w$. Note also that $A$ is the set of roll-calls for which $a$ is pivotal in the game $v \wedge w$, while $A, B, C$ form a partition of the set of roll-calls in which $a$ is pivotal in the game $v \vee w$. For any $a \in N$ we have

$$
\begin{aligned}
\phi_{a}(v) & =\frac{|A|+|B|}{3^{n} n !} \quad \phi_{a}(w)=\frac{|A|+|C|}{3^{n} n !} \\
\phi_{a}(v \vee w) & =\frac{|A|+|B|+|C|}{3^{n} n !} \quad \phi_{a}(v \wedge w)=\frac{|A|}{3^{n} n !} .
\end{aligned}
$$

Thus $\phi(v \vee w)+\phi(v \wedge w)=\phi(v)+\phi(w)$ and the Shapley-Shubik index satisfies the transfer axiom.

Efficiency In every roll-call there is one and only one player that is pivotal, from the definition of the Shapley-Shubik index in (1), we get that it satisfies efficiency.

As it is well-known these axioms for simple games are independent and they fully characterized the Shapley-Shubik index for simple games. This is not true for $(3,2)$-simple games. For instance, let $\bar{\phi}$ be the standard Shapley-Shubik index for simple games. Then consider the index $\varphi$ for $(3,2)$-simple games defined as $\varphi(v)=\bar{\phi}(V)$ where $V$ is the simple game associated to the (3,2)-simple game $v$ and defined as

$$
V(S)=1 \text { if and only if } v(S, N \backslash S, \emptyset)=1 .
$$

Then $\varphi$ satisfies the anonymity, null player, transfer and efficiency axioms for (3,2)-simple games since the Shapley-Shubik index satisfies them on simple games, but it is different from $\phi$, for instance $\varphi\left(u_{(a, b, \emptyset)}\right)=(1,0)$ while $\phi\left(u_{(a, b, \emptyset)}\right)=\left(\frac{2}{3}, \frac{1}{3}\right)$. 
The Banzhaf index for (3,2)-simple games satisfies the anonymity, the null player, the transfer and the Banzhaf total power axioms.

Let us consider the different properties.

Anonymity The Banzhaf index for (3,2)-simple games satisfies anonymity: actually if $a \in N$ and $a$ is yes-no swinger for tripartition $S$, then it is clear that $\pi a$ is a yes-no swinger for $\pi S$.

Null player If $a$ is a null player in the game $v$, then $v(S)=v\left(S_{\downarrow \downarrow a}\right)$ for all $S \in 3^{N}$ such that $a \in S_{1}$. So $\beta_{a}(v)=0$.

Transfer Let $v$ and $w$ be two (3,2)-simple games and $V$ and $W$ be the set of their winning tripartitions, then consider the following sets:

$$
\begin{aligned}
& A=\left\{S \in 3^{N}: a \in S_{1}, S \in V \backslash W, S_{\downarrow \downarrow a} \notin V\right\} \\
& B=\left\{S \in 3^{N}: a \in S_{1}, S \in W \backslash V, S_{\downarrow \downarrow a} \notin W\right\} \\
& C=\left\{S \in 3^{N}: a \in S_{1}, S \in V \cap W, S_{\downarrow \downarrow a} \in W \backslash V\right\} \\
& D=\left\{S \in 3^{N}: a \in S_{1}, S \in V \cap W, S_{\downarrow \downarrow a} \in V \backslash W\right\} \\
& E=\left\{S \in 3^{N}: a \in S_{1}, S \in V \cap W, S_{\downarrow \downarrow a} \notin V \cap W\right\} .
\end{aligned}
$$

Note that the sets $A, C$ and $E$ form a partition for the set of yes-no swings of $a$ in $v ; B, D$ and $E$ form a partition for the set of yes-no swings of $a$ in $w$. All the five sets form the set of yes-no swings for player $a$ in $v \vee w$, while $E$ is the set of swings for player $a$ in $v \wedge w$. So the Banzhaf index, which counts the number of yes-no swings, satisfies the transfer axiom.

Banzhaf total power This axiom is trivially satisfied from the definition of the Banzhaf index.

Again, anonymity, null player, transfer and Banzhaf total power are independent axioms on simple games, but the Banzhaf index for $(3,2)$-games is not uniquely determined using only these four. For instance, let $\bar{\beta}$ be the standard Banzhaf index for simple games, consider the index $\varphi$ for $(3,2)$-simple games defined as $\varphi(v)=\bar{\beta}(V)$ where $V$ is the simple game associated to the game $v$ and defined as $V(S)=1$ if and only if $v(S, N \backslash S, \emptyset)=1$. Then $\varphi$ satisfies the anonymity, null player, transfer and Banzhaf total power axioms since the Banzhaf index satisfies them on simple games, but it is different from $\beta$, for instance $\varphi\left(u_{(a, b, \emptyset)}\right)=(1,0)$ while $\beta\left(u_{(a, b, \emptyset)}\right)=\left(\frac{2}{3}, \frac{1}{3}\right)$.

As we have seen, these classical axioms are not enough to characterize the Shapley-Shubik and the Banzhaf indices on the space of (3,2)-simple games. We are going to propose two new axioms in order to uniquely determine these two indices.

\subsection{A new axiom for the Shapley-Shubik index for $(3,2)-$ simple games}

Let us introduce another index for $(3,2)$-simple games, that we use to state the new axiom for the Shapley-Shubik index for (3,2)-simple games. Given $v \in \mathfrak{T}^{N}$, 
let $\mathcal{W}(v)$ be the set of its winning coalitions. Then the structural decisiveness index is defined as the map $\delta: \mathfrak{T}^{N} \rightarrow \mathbb{R}$

$$
\delta(v)=3^{-n}|\mathcal{W}(v)| .
$$

This index is assumed to provide a measure of the formal effectiveness of the game to pass decisions without taking into account either the personality of concrete agents or their preferences as to a particular proposal versus the status quo. For example, let us consider the unanimity game $u_{S}$ with $S=\left(S_{1}, S_{2}, S_{3}\right)$ and $a \in S_{1}$; then

$$
\delta\left(u_{S}\right)=\frac{2^{s_{2}}}{3^{s_{1}+s_{2}}} \quad \delta\left(u_{S \downarrow a}\right)=2 \delta\left(u_{S}\right) \quad \delta\left(u_{S \downarrow \downarrow a}\right)=3 \delta\left(u_{S}\right) .
$$

We can now discuss the axiom we have to introduce to describe the behavior of the index on unanimity game.

Yes-abstain loss on unanimity games An index $\psi$ satisfies the yes-abstain loss on unanimity games if for any tripartition $S \in 3^{N}$ with $a \in S_{1}$ it holds:

$$
\psi_{a}\left(u_{S}\right)-\psi_{a}\left(u_{S \downarrow a}\right)=\psi_{a}\left(u_{S \downarrow a}\right)-\frac{\delta\left(u_{S \downarrow \downarrow a}\right)}{s_{1}+s_{2}} .
$$

In the following, we denote with $f\left(u_{S}\right)=\frac{\delta\left(u_{S \downarrow \downarrow a}\right)}{s_{1}+s_{2}}$, so that the previous equation can also be written as

$$
\psi_{a}\left(u_{S}\right)-\psi_{a}\left(u_{S \downarrow a}\right)=\psi_{a}\left(u_{S \downarrow a}\right)-f\left(u_{S}\right) .
$$

In order to prove that the Shapley-Shubik index for $(3,2)$-simple games satisfies the yes-abstain loss on unanimity games, we have to focus on unanimity games. In particular, from now on, we fix a tripartition $S$ with $a \in S_{1}$ and consider the game $u_{S}$. We want to compute $\phi_{a}\left(u_{S}\right)$ and then compare it with $\phi_{a}\left(u_{S \downarrow a}\right)$.

Let $u_{S}$ be a unanimity game, then player $a \in S_{1}$ is pivotal in the roll-call $\mathcal{R} \in \mathcal{R}_{a}^{n o}$ if and only if $a$ is pivotal in the roll-call $\mathcal{R}_{\uparrow a} \in \mathcal{R}_{a}^{a b s}$.

A roll-call is winning in the game $u_{S}$ if and only if all players belonging to $S_{1}$ are voting "yes" and all players belonging to $S_{2}$ are not voting "no".

If $a \in S_{1}$ is pivotal by voting "no" in the roll-call $\mathcal{R}$, then the outcome of $\mathcal{R}$ is negative. The roll-call $\mathcal{R}_{\downarrow a}$ represents the same situation of $\mathcal{R}$ with the only difference that player $a$ abstains instead of voting no. But $a$ is still pivotal abstaining and fixing as negative the outcome of the roll-call.

Analogously, if player $a$ is pivotal by abstaining, in the same situation $a$ is also pivotal by voting "no".

Lemma 3.2 implies that $\left|A_{a, u_{S}}\right|=\left|N_{a, u_{S}}\right|$, but also $\left|A Y_{a, u_{S}}\right|=\left|N Y_{a, u_{S}}\right|$ and $\left|A \bar{Y}_{a, u_{S}}\right|=\left|N \bar{Y}_{a, u_{S}}\right|$, because of the one-to-one correspondence among each pair of the sets $\mathcal{R}_{a}^{\text {yes }}, \mathcal{R}_{a}^{a b s}$, and $\mathcal{R}_{a}^{n o}$. 
Let $u_{S}$ be a unanimity game, if player $a \in S_{1}$ is pivotal in the roll-call $\mathcal{R} \in \mathcal{R}_{a}^{\text {yes }}$, then $a$ is pivotal in the roll-call $\mathcal{R}_{\downarrow a} \in \mathcal{R}_{a}^{a b s}$ and in the roll-call $\mathcal{R}_{\downarrow \downarrow a} \in \mathcal{R}_{a}^{n o}$

A roll-call is winning in the game $u_{S}$ if and only if all players belonging to $S_{1}$ are voting "yes" and all players belonging to $S_{2}$ are not voting "no".

If $a \in S_{1}$ is pivotal in the roll-call $\mathcal{R}$ by voting "yes", this means that after $a$ 's vote the outcome is positive and all the other players in $S_{1}$ and $S_{2}$ voted before $a$. This also means that after $a$ only some of the players belonging to $S_{3}$ are going to vote, but they are null players and can not be pivotal.

In the roll-call $\mathcal{R}, a$ is the last player who has the power to change the outcome of the game, thus $a$ is pivotal also in $\mathcal{R}_{\downarrow \downarrow a}$ voting "no" and in $\mathcal{R}_{\downarrow a}$ abstaining.

The converse is not true. For instance consider the tripartition $S=(a, b, c)$ and the game $u_{S}$. In any roll-call in which $a$ is the first to vote, he is pivotal abstaining or voting "no". On the other hand if $a$ votes "yes" as first player, then $b$ is pivotal: if she votes "no" the outcome is negative, while if she abstains or votes "yes" the outcome is positive.

Note that by definition $\left|A Y_{a, u_{S}}\right| \leq\left|Y_{a, u_{S}}\right|$ and $\left|N Y_{a, u_{S}}\right| \leq\left|Y_{a, u_{S}}\right|$. Lemma 3.2 implies that $\left|Y_{a, u_{S}}\right| \leq\left|A Y_{a, u_{S}}\right|$ and $\left|Y_{a, u_{S}}\right| \leq\left|N Y_{a, u_{S}}\right|$. Thanks to these considerations and Lemma 3.2 we have $\left|Y_{a, u_{S}}\right|=\left|A Y_{a, u_{S}}\right|=\left|N Y_{a, u_{S}}\right|$.

Hence, from the previous remarks, the Shapley-Shubik index on the unanimity (3,2)-simple game $u_{S}$ of player $a \in S_{1}$ is

$$
\begin{aligned}
\phi_{a}\left(u_{S}\right) & =\frac{1}{3^{n} n !}\left(\left|Y_{a, u_{S}}\right|+\left|A_{a, u_{S}}\right|+\left|N_{a, u_{S}}\right|\right) \\
& =\frac{1}{3^{n} n !}\left(\left|Y_{a, u_{S}}\right|+\left|A Y_{a, u_{S}}\right|+\left|A \bar{Y}_{a, u_{S}}\right|+\left|N Y_{a, u_{S}}\right|+\left|N \bar{Y}_{a, u_{S}}\right|\right) \\
& =\frac{1}{3^{n} n !}\left(3\left|Y_{a, u_{S}}\right|+2\left|N \bar{Y}_{a, u_{S}}\right|\right)
\end{aligned}
$$

It is possible to calculate the value $\left|Y_{a, u_{S}}\right|$ thanks to the following lemma.

A player $a \in S_{1}$ is pivotal in the game $u_{S}$ for the roll-call $\mathcal{R} \in \mathcal{R}_{a}^{\text {yes }}$ if and only if all players in $\left(S_{1} \backslash\{a\}\right) \cup S_{2}$ are before him in $q R$, they vote "yes" if they belong to $S_{1}$, and they vote "yes" or abstain if they belong to $S_{2}$.

In particular

$$
\left|Y_{a, u_{S}}\right|=2^{s_{2}} 3^{s_{3}} \frac{\left(s_{1}+s_{2}+s_{3}\right) !}{s_{1}+s_{2}}
$$

A roll-call is winning in the game $u_{S}$ if and only if all players belonging to $S_{1}$ are voting "yes" and all players belonging to $S_{2}$ are not voting "no". Thus if a player $a \in S_{1}$ is pivotal in the roll-call $\mathcal{R} \in \mathcal{R}_{a}^{\text {yes }}$, he is the last player of the set $S_{1} \cup S_{2}$ to vote and he is the last player that has the possibility to influence the outcome and fix it as positive. All the players before him were not pivotal, so they voted "yes" if they belong to $S_{1}$, they abstained or voted "yes" if they belong to $S_{2}$.

To prove the second part of the thesis, we have to count the number of roll-calls $\mathcal{R} \in Y_{a, u_{S}}$. Actually, if $j=0, \ldots, s_{3}$, player $a$ can vote $j$ positions 


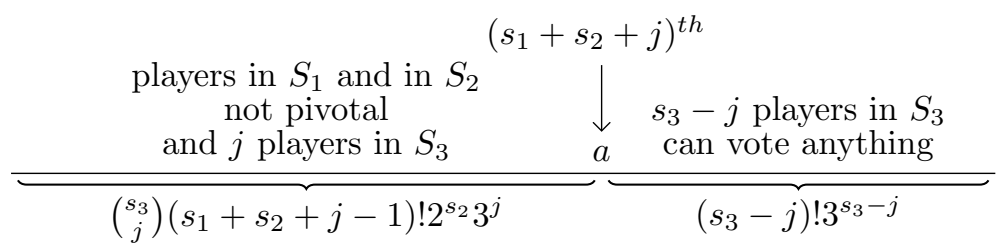

Figure 1: Roll-calls in which $a \in S_{1}$ is pivotal by voting "yes"

after that all the players in $S_{1} \backslash\{a\} \cup S_{2}$ voted. This means that $j$ players belonging to $S_{3}$ vote before $a$ and $s_{3}-j$ players belonging to $S_{3}$ vote after $a$. There are $\left(\begin{array}{c}s_{3} \\ j\end{array}\right)$ different ways to choose the $j$ players, then $\left(s_{1}+s_{2}+j-1\right) ! 2^{s_{2}} 3^{j}$ possibilities for the players before $a$ and $\left(s_{3}-j\right) ! 3^{s_{3}-j}$ for the players after $a$. Hence,

$$
\begin{aligned}
\left|Y_{a, u_{S}}\right| & =\sum_{j=0}^{s_{3}}\left(\begin{array}{c}
s_{3} \\
j
\end{array}\right)\left(s_{1}+s_{2}+j-1\right) ! 2^{s_{2}} 3^{j}\left(s_{3}-j\right) ! 3^{s_{3}-j} \\
& =2^{s_{2}} 3^{s_{3}} \frac{\left(s_{1}+s_{2}+s_{3}\right) !}{s_{1}+s_{2}} .
\end{aligned}
$$

We can now discuss how the power of player $a$ changes when $a$ decreases the support of one level. We evaluate the Shapley-Shubik index for $(3,2)$-simple games of player $a \in S_{1}$ in the unanimity game $u_{S \downarrow a}$, generated by the tripartition $\left(S_{1} \backslash\{a\}, S_{2} \cup\{a\}, S_{3}\right)$.

Firstly note that if $a$ is pivotal by abstaining in $u_{S \downarrow a}$, then in the same situation $a$ is pivotal also by voting "yes"; this means that $\left|A \bar{Y}_{a, u_{S \downarrow}}\right|=0$. Then observe that if $\mathcal{R} \notin A \bar{Y}_{a, u_{S}}$ and $a$ is pivotal in $\mathcal{R}$ for $u_{S}$, then $a$ is pivotal in $\mathcal{R}$ also for $u_{S \downarrow a}$. This means that $Y_{a, u_{S \downarrow a}}=Y_{a, u_{S}}$ and

$$
\begin{array}{rlrl}
A Y_{a, u_{S \downarrow a}} & =A Y_{a, u_{S}} & & A \bar{Y}_{a, u_{S \downarrow a}}=\emptyset \\
N Y_{a, u_{S \downarrow a}} & =N Y_{a, u_{S}} & N \bar{Y}_{a, u_{S \downarrow a}}=N \bar{Y}_{a, u_{S}} .
\end{array}
$$

Hence,

$$
\phi_{a}\left(u_{S \downarrow a}\right)=\frac{1}{3^{n} n !}\left(3\left|Y_{a, u_{S}}\right|+\left|N \bar{Y}_{a, u_{S}}\right|\right) .
$$

Finally, to establish how the index changes when a player switches from voting "yes" to abstaining in a unanimity game, we compare equations (3) and (4) and get the following:

$$
\phi_{a}\left(u_{S}\right)-\phi_{a}\left(u_{S \downarrow a}\right)=\frac{1}{3^{n} n !}\left|N \bar{Y}_{a, u_{S}}\right|
$$

To compare the difference with something we can explicit, we also get the following equality

$$
2 \phi_{a}\left(u_{S \downarrow a}\right)-\phi_{a}\left(u_{S}\right)=\frac{3}{3^{n} n !}\left|Y_{a, u_{S}}\right|
$$


that can be re-written as

$$
\phi_{a}\left(u_{S}\right)-\phi_{a}\left(u_{S \downarrow a}\right)=\phi_{a}\left(u_{S \downarrow a}\right)-\frac{3}{3^{n} n !}\left|Y_{a, u_{S}}\right| .
$$

From this result and Lemma 3.2 we obtain the following.

The Shapley-Shubik index satisfies the yes-abstain loss on unanimity games.

\section{The characterization of the Shapley-Shubik index for $(3,2)$-simple games}

We can now state the main theorem to characterize the Shapley-Shubik index for $(3,2)$-simple games. We also prove the independence of the five axioms we are going to use, so all of them are necessary in order to uniquely characterize the Shapley-Shubik power index for $(3,2)$-simple games.

[Shapley-Shubik index for (3,2)-simple games] Let $\psi: \mathfrak{T}^{N} \rightarrow \mathbb{R}^{n}$ be an index for $(3,2)$-simple games, then $\psi$ satisfies the anonymity, null player, transfer, efficiency and yes-abstain loss on unanimity games axioms if and only if $\psi$ is the Shapley-Shubik index for $(3,2)$-simple games.

In Lemma 3.1 and in Proposition 3.2 it is proved that the Shapley-Shubik index for (3,2)-simple games satisfies all the axioms, we just need to prove that only one index satisfies all of them.

So, let $\psi$ be an index that satisfies the hypothesis. We will prove that it is uniquely determined on a game $v$, using induction on the number of minimal winning tripartitions of $v$.

First, suppose that $\left|\mathcal{W}^{m}(v)\right|=1$. Then $v=u_{S}$ for some tripartition $S \in 3^{N}$ and $S \neq(\emptyset, \emptyset, N)$. We again use induction on the number of elements in $S_{2}$.

$\left|S_{2}\right|=0$ Then $S=\left(S_{1}, \emptyset, N \backslash S_{1}\right)$ for some $S_{1} \subseteq N$. All players in $S_{3}=N \backslash S_{1}$ are null players, so if $c \in S_{3}: \psi_{c}\left(u_{S}\right)=0$, on the other hand all players in $S_{1}$ have the same role, thus, thanks to the anonymity and efficiency axioms we have $\psi_{a}\left(u_{S}\right)=\frac{1}{s_{1}}$, for any $a \in S_{1}$.

$\left|S_{2}\right|=t+1$ Suppose now that the thesis is true for any tripartition $T$ such that $\left|T_{2}\right| \leq$ $t$, we want to prove it for a tripartition $S$ such that $\left|S_{2}\right|=t+1$. Given the tripartition $S=\left(S_{1}, S_{2}, S_{3}\right)$, there exist a player $p \in S_{2}$ and a tripartition $T=\left(T_{1}, T_{2}, T_{3}\right)$ such that $T_{\downarrow p}=S$ and $\left|T_{2}\right|=t$. Since $\psi$ satisfies the yes-abstain loss on unanimity games:

$$
\psi_{p}\left(u_{S}\right)=\psi_{p}\left(u_{T \downarrow p}\right)=\frac{1}{2}\left[\psi_{p}\left(u_{T}\right)+f\left(u_{T}\right)\right]
$$

then the induction hypothesis and anonymity imply that $\psi_{b}\left(u_{S}\right)$ is uniquely determined for all players $b \in S_{2}$.

Thanks to anonymity and efficiency:

$$
s_{1} \psi_{a}\left(u_{S}\right)+s_{2} \psi_{b}\left(u_{S}\right)=1,
$$


so we can determine $\psi_{a}\left(u_{S}\right)$ for $a \in S_{1}$. All players in $S_{3}$ are null, so $\psi_{c}\left(u_{S}\right)=0$ if $c \in S_{3}$.

Thus, $\psi$ coincides with the Shapley-Shubik power index for $(3,2)$-simple games for any unanimity game $u_{S}$.

Now, suppose that the thesis holds for any game $v$ such that $\left|\mathcal{W}^{m}(v)\right| \leq k-1$; we need to prove it for $v$ such that $\left|\mathcal{W}^{m}(v)\right|=k$.

If $\mathcal{W}^{m}(v)=\left\{S^{1}, \ldots, S^{k}\right\}$, then $v=u_{S^{1}} \vee u_{S^{2}} \vee \cdots \vee u_{S^{k}}$, since $\psi$ satisfies the transfer axiom:

$$
\psi(v)=\psi\left(u_{S^{1}}\right)+\psi\left(u_{S^{2}} \vee \cdots \vee u_{S^{k}}\right)-\psi\left(u_{S^{1}} \wedge u_{S^{2}} \wedge \cdots \wedge u_{S^{k}}\right) .
$$

The conjunction of unanimity games is still a unanimity game, so all games in the right-hand side of the previous equation have a number of minimal winning tripartitions smaller than $\left|W^{m}(v)\right|$. Using the induction hypothesis, $\psi$ coincides with the Shapley-Shubik index for all of them and this ends the proof.

Now, that we proved that the Shapley-Shubik power index for games with abstention is uniquely characterized by the five axioms we can show how these axioms allow to compute the index for every unanimity game by means of a recursive procedure.

Consider the unanimity game $u_{S}$ with the set $S_{3}$ of people voting no. Then consider the game $u_{T^{0}}$ where $T^{0}=\left(\emptyset, N \backslash S_{3}, S_{3}\right)$. For every player $a \in S_{3}$ thanks to null player we have $\phi_{a}\left(u_{T^{0}}\right)=0$, for every $a \in N \backslash S_{3}$ thanks to anonymity and efficiency it holds

$$
\phi_{a}\left(u_{T}\right)=\frac{1}{n-s_{3}} .
$$

Then consider the game $u_{T^{1}}$ where $\left.T^{1}=\left(p, N \backslash\left(S_{3} \cup p\right), S_{3}\right)\right)$ for some player $p \in S_{1}$. Thanks to (2) we can compute $\phi_{p}\left(u_{T^{1}}\right)$, then for any $a \neq p \in N \backslash S_{3}$, $\phi_{a}\left(u_{T^{1}}\right)$ can be computed using efficiency and players in $S_{3}$ are still null players.

It is clear that this process can be reiterated until we reach the games $u_{S}$ and establish the value for all players in $S_{1}$ and $S_{2}$ using the yes-abstain loss on unanimity games and efficiency.

\subsection{Independence of the axiom for the Shapley-Shubik power index for $(3,2)$-simple games}

The five axioms for $(3,2)$-simple games are independent. We are going to give examples of power indices for $(3,2)$-simple games that satisfy only four of them, as summarized in Table 2 .

Not anonymity Consider the index $\psi^{1}$ defined on unanimity games as follows.

- If $s_{3}=n-2$ then for any two players $a$ and $b$ such that $a<b$,

- if $S=(a b, \emptyset, N \backslash\{a, b\})$, then

$$
\psi_{a}^{1}\left(u_{S}\right)=\frac{1}{2}+\varepsilon, \quad \psi_{b}^{1}\left(u_{S}\right)=\frac{1}{2}-\varepsilon
$$




\begin{tabular}{|c|c|c|c|c|c|}
\hline & Anonymity & Null & Transfer & Efficiency & New axiom \\
\hline$\psi^{1}$ & - & $\checkmark$ & $\checkmark$ & $\checkmark$ & $\checkmark$ \\
$\psi^{2}$ & $\checkmark$ & - & $\checkmark$ & $\checkmark$ & $\checkmark$ \\
$\psi^{3}$ & $\checkmark$ & $\checkmark$ & - & $\checkmark$ & $\checkmark$ \\
$\psi^{4}$ & $\checkmark$ & $\checkmark$ & $\checkmark$ & - & $\checkmark$ \\
$\varphi$ & $\checkmark$ & $\checkmark$ & $\checkmark$ & $\checkmark$ & - \\
\hline
\end{tabular}

Table 2: Independence of the axioms that characterize the Shapley-Shubik index for (3,2)-simple games

- if $S=(a, b, N \backslash\{a, b\})$, then

$$
\psi_{a}^{1}\left(u_{S}\right)=\frac{2}{3}+\frac{\varepsilon}{2}, \quad \psi_{b}^{1}\left(u_{S}\right)=\frac{1}{3}-\frac{\varepsilon}{2}
$$

- if $S=(b, a, N \backslash\{a, b\})$, then

$$
\psi_{a}^{1}\left(u_{S}\right)=\frac{1}{3}+\frac{\varepsilon}{2}, \quad \psi_{b}^{1}\left(u_{S}\right)=\frac{2}{3}-\frac{\varepsilon}{2}
$$

- if $S=(\emptyset, a b, N \backslash\{a, b\})$, then

$$
\psi_{a}^{1}\left(u_{S}\right)=\frac{1}{2}+\frac{\varepsilon}{4}, \quad \psi_{b}^{1}\left(u_{S}\right)=\frac{1}{2}-\frac{\varepsilon}{4}
$$

where $\varepsilon>0$;

- if $s_{3} \neq n-2, \psi^{1}\left(u_{S}\right)=\phi\left(u_{S}\right)$ where $\phi$ is the Shapley-Shubik index for $(3,2)$-simple games.

Then extend $\psi^{1}$ to $\mathfrak{T}^{N}$ using transfer.

It is clear that this index satisfies null player and efficiency. It also satisfies the yes-abstain loss on unanimity games, because it coincides with the ShapleyShubik index for (3,2)-simple games when $s_{3} \neq n-2$ and if $s_{3}=n-2$ the yes-abstain loss on unanimity games is satisfied by the definition of $\psi^{1}$, as it is easy to check. However, $\psi^{1}$ does not satisfy anonymity, because for instance

$$
\psi_{a}^{1}\left(u_{(a b, \emptyset, N \backslash\{a, b\})}\right)-\psi_{b}^{1}\left(u_{(a b, \emptyset, N \backslash\{a, b\})}\right)=2 \varepsilon \neq 0 .
$$

Not null player Consider the index $\psi^{2}$ defined on unanimity games as follows.

- If $S=(\emptyset, a, N \backslash\{a\})$ for some $a \in N$, then

$$
\psi_{a}^{2}\left(u_{S}\right)=1-\varepsilon, \quad \psi_{b}^{2}\left(u_{S}\right)=\frac{\varepsilon}{n-1}
$$

for any $b \neq a$, with $\varepsilon>0$; 
- if $S=(a, \emptyset, N \backslash\{a\})$ for some $a \in N$, then

$$
\psi_{a}^{2}\left(u_{S}\right)=1-2 \varepsilon, \quad \psi_{b}^{2}\left(u_{S}\right)=\frac{2 \varepsilon}{n-1}
$$

for any $b \neq a$, with $\varepsilon>0$;

- for any other $S \in 3^{N}, \psi^{2}\left(u_{S}\right)=\phi\left(u_{S}\right)$ where $\phi$ is the Shapley-Shubik index for $(3,2)$-simple games.

Then extend $\psi^{2}$ to $\mathfrak{T}^{N}$ using transfer.

It is clear that this index satisfies anonymity and efficiency. It also satisfies the yes-abstain loss on unanimity games since it coincides with the Shapley-Shubik index on unanimity games such that $s_{3} \neq n-1$ and if $s_{3}=n-1$ and $a \in S_{1}$

$$
2 \psi^{2}\left(u_{S \downarrow a}\right)-\psi^{2}\left(u_{S}\right)=1=f\left(u_{(a, \emptyset, N \backslash\{a\})}\right) .
$$

However, $\psi^{2}$ does not satisfy null player: any $b \neq a$ is a null player in the game $u_{(\emptyset, a, N \backslash\{a\})}$ but $\psi_{b}^{2}\left(u_{(\emptyset, a, N \backslash\{a\})}\right)=\frac{\varepsilon}{n-1} \neq 0$.

Not transfer Consider the index $\psi^{3}$ defined as $\psi^{3}\left(u_{S}\right)=\phi\left(u_{S}\right)$ for any unanimity game $u_{S}$ and for any other game $v$

$$
\psi_{a}^{3}(v)= \begin{cases}0 & \text { if } a \text { is a null player } \\ \frac{1}{k} & \text { otherwise }\end{cases}
$$

where $k=\mid\{p \in N: p$ is not a null player in $v\} \mid$.

The index $\psi^{3}$ satisfies the null player, the anonymity, and the efficiency axioms; it also satisfies the yes-abstain loss on unanimity games, since it coincides with the Shapley-Shubik index on unanimity games. From the definition of $\psi^{3}(v)$ it is clear that this index does not satisfy the transfer axiom.

Not efficiency Consider the index $\psi^{4}$ defined on unanimity game as

$$
\psi_{a}^{4}\left(u_{S}\right)= \begin{cases}1 & \text { if } a \in S_{1} \\ \frac{1}{2}+\frac{2^{s_{2}-1}}{3^{s_{1}+s_{2}-1}\left(s_{1}+s_{2}\right)} & \text { if } a \in S_{2} \\ 0 & \text { if } a \in S_{3}\end{cases}
$$

and extended to $\mathfrak{T}^{N}$ using transfer. Then $\psi^{4}$ satisfies anonymity, null player and transfer. It also satisfies the yes-abstain loss on unanimity games since for any tripartition $S$ with $a \in S_{1}$ :

$$
\begin{aligned}
2 \psi_{a}^{4}\left(u_{S \downarrow a}\right) & =2\left[\frac{1}{2}+\frac{2^{s_{2}-1}}{3^{s_{1}+s_{2}-1}\left(s_{1}+s_{2}\right)}\right] \\
& =1+\frac{2^{s_{2}}}{3^{s_{1}+s_{2}-1}\left(s_{1}+s_{2}\right)} \\
& =\psi_{a}^{4}\left(u_{S}\right)+f\left(u_{S}\right)
\end{aligned}
$$


However, $\psi^{4}$ does not satisfy efficiency, for instance $\psi^{4}\left(u_{(N, \emptyset, \emptyset)}\right)=(1, \ldots, 1)$ so that $\sum_{a \in N} \psi^{4}\left(u_{(N, \emptyset, \emptyset)}\right)=n \neq 1$.

Not yes-abstain loss on unanimity games As we examined in Remark 3.1 , the index $\varphi$, that is Shapley-Shubik index for simple games computed on the simple games associated to the game with abstention, satisfies null player, anonymity, transfer, and efficiency, but does not satisfy the yes-abstain loss on unanimity games.

\section{A similar approach for the Banzhaf index for $(3,2)$-simple games}

We now consider the Banzhaf index for (3,2)-simple games and characterize this index, as we did in the previous sections with the Shapley-Shubik index for $(3,2)$-simple games. Let us start with a preliminary lemma that shows how simple it is to compute the Banzhaf index for (3,2)-simple games on unanimity games. This lemma will be used in the following to prove the new axioms we are going to introduce in order to characterize the Banzhaf index for (3,2)-simple games.

Let $S \neq(\emptyset, \emptyset, N)$ be a tripartition of $N$, then the Banzhaf index for $(3,2)$ simple games on the unanimity games $u_{S}$ is

$$
\beta_{p}\left(u_{S}\right)= \begin{cases}3 \delta\left(u_{S}\right) & \text { if } p \in S_{1} \\ \frac{3}{2} \delta\left(u_{S}\right) & \text { if } p \in S_{2} \\ 0 & \text { if } p \in S_{3}\end{cases}
$$

From the previous lemma, it follows, in particular, that if $a \in S_{1}$ and $b \in S_{2}$, it holds

$$
\beta_{a}\left(u_{S}\right)=2 \beta_{b}\left(u_{S}\right) .
$$

We have to compute $\eta_{a}\left(u_{S}\right)$ for any player $a \in N$. Assume first that $a \in S_{1}$. Remember that $u_{S}(T)=1$ if and only if $S_{1} \subseteq T_{1}$ and $S_{2} \subseteq T_{1} \cup T_{2}$. Note that if $a \in S_{1} \cap T_{1}$, the condition $u_{S}(T)=1$ implies $u_{S}\left(T_{\downarrow \downarrow a}\right)=0$. Moreover, if $a \in S_{1}$, the conditions $a \in T_{1}$ and $u_{S}(T)=1$ are equivalent to $S \subseteq T$. Hence,

$$
\begin{aligned}
\eta_{a}\left(u_{S}\right) & =\left|\left\{T \in 3^{N}: a \in T_{1}, u_{S}(T)-u_{S}\left(T_{\downarrow \downarrow a}\right)=1\right\}\right| \\
& =\left|\left\{T \in 3^{N}: a \in T_{1}, u_{S}(T)=1\right\}\right| \\
& =\left|\left\{T \in 3^{N}: S \subseteq T\right\}\right|=2^{s_{2}} 3^{s_{3}} .
\end{aligned}
$$

Suppose now $a \in S_{2}$. Analogously we have

$$
\eta_{a}\left(u_{S}\right)=\mid\left\{T \in 3^{N}: a \in T_{1} \text { and } S \subseteq T\right\} \mid=2^{s_{2}-1} 3^{s_{3}} .
$$

Finally, assume $a \in S_{3}$. Players in $S_{3}$ are null, so $\beta_{a}\left(u_{S}\right)=0$. 
Since the Banzhaf index for $(3,2)$-simple games is given by

$$
\beta_{a}\left(u_{S}\right)=\frac{\eta_{a}\left(u_{S}\right)}{3^{n-1}}
$$

and $n=s_{1}+s_{2}+s_{3}$, we have the thesis.

In the [Dubey and Shapley [1979]] characterization of the Banzhaf power index for simple games, the Banzhaf total power axiom is introduced in order to replace efficiency, that is used for the Shapley-Shubik power index. However, the Banzhaf total power axiom is not a convincing axiom; some subsequent axiomatic characterization of the Banzhaf power index avoided this axiom, see for instance [Laurelle and Valenciano [2001], Lehrer [1988], Albizuri [2001]].

We want to follow the classical approach and use the same set of axioms to characterize the Shapley-Shubik and the Banzhaf indices for $(3,2)$-simple games. However, we will replace the Banzhaf total power with a weaker condition that refers only to unanimity games.

Total power on unanimity games An index $\psi$ satisfies the total power on unanimity games if for any tripartition $S \neq(\emptyset, \emptyset, N)$

$$
\sum_{a \in N} \psi_{a}\left(u_{S}\right)=\frac{3}{2}\left(2 s_{1}+s_{2}\right) \delta\left(u_{S}\right)
$$

The Banzhaf index for (3,2)-simple games satisfies the total power on unanimity games axiom.

The thesis follows from anonymity and Lemma 5.

As we have previously done for the Shapley-Shubik index on $(3,2)$-simple games, it is necessary to add another axiom in order to uniquely characterize the Banzhaf index on (3,2)-simple games. The new axiom defined in equation (2), describes what a player is losing when passing from voting "yes" to abstaining; we introduce the following axiom to describe a different idea: the power of player $a$ does not change in the game $u_{S}$ and in $u_{S \downarrow a}$.

Yes-abstain null loss on unanimity games An index $\psi$ satisfies the yesabstain null loss axiom if for any unanimity game $u_{S}$ and $a \in S_{1}$ it holds

$$
\psi_{a}\left(u_{S}\right)=\psi_{a}\left(u_{S \downarrow a}\right) .
$$

We immediately have the following result. Consider the unanimity game $u_{S}$ with $a \in S_{1}$. Then the Banzhaf index on $(3,2)$-simple games satisfies the yesabstain null loss axiom and in particular

$$
\beta_{a}\left(u_{S}\right)=\beta_{a}\left(u_{S \downarrow a}\right) .
$$




\subsection{Characterization of the Banzhaf index for $(3,2)$-simple games}

Let $\psi: \mathfrak{T}^{N} \rightarrow \mathbb{R}^{n}$ be an index for $(3,2)$-simple games, then $\psi$ satisfies anonymity, null player, transfer, total power on unanimity games and yes-abstain null loss if and only if $\psi$ is the Banzhaf index for $(3,2)$-simple games.

We already proved that the Banzhaf index for (3,2)-simple games satisfies all these properties, so we just need to prove that if $\psi$ is a power index that satisfies the hypothesis, then it is uniquely determined. We use induction on the number of minimal winning tripartitions of the game $v$.

Suppose that $\left|\mathcal{W}^{m}(v)\right|=1$, then $v=u_{S}$ for some tripartition $S$. So we start proving that $\psi$ coincides with the Banzhaf index on unanimity games.

We again use induction, this time on the cardinality of $S_{2}$.

$\left|S_{2}\right|=0$. Then $S=\left(S_{1}, \emptyset, N \backslash S_{1}\right)$ for some $S_{1} \subseteq N$. Then players in $S_{3}=N \backslash S_{1}$ are null, so $\psi_{c}\left(u_{S}\right)=0$ for all $c \in S_{3}$. Players in $S_{1}$ are symmetric and thanks to anonymity and total power on unanimity game, if $a \in S_{1}$

$$
\beta_{a}\left(u_{\left(S_{1}, \emptyset, N \backslash S_{1}\right)}\right)=\frac{1}{s_{1}} \frac{2 s_{1} 2^{-1}}{3^{s_{1}-1}}=\frac{1}{3^{s_{1}-1}} .
$$

So, $\psi$ is uniquely determined on unanimity games with $s_{2}=0$ and it coincides with the Banzhaf index for $(3,2)$-simple games.

$\left|S_{2}\right|=t+1$. Suppose now that the thesis is true for any tripartition $T$ such that $\left|T_{2}\right| \leq$ $t$, we want to prove this for a tripartition $S$ such that $\left|S_{2}\right|=t+1$. Given a tripartition $S=\left(S_{1}, S_{2}, S_{3}\right)$ such that $\left|S_{2}\right|=t+1$, there exist a player $p \in S_{2}$ and a tripartition $T=\left(T_{1}, T_{2}, T_{3}\right)$ such that $T_{\downarrow p}=S$ and $T_{2}=t$. Since $\psi$ satisfies yes-abstain null loss:

$$
\psi_{p}\left(u_{S}\right)=\psi_{p}\left(u_{T \downarrow p}\right)=\psi_{p}\left(u_{T}\right),
$$

then the induction hypothesis and anonymity imply that $\psi_{b}\left(u_{S}\right)$ is uniquely determined for all players $b \in S_{2}$.

Using again anonymity and the total power on unanimity game:

$$
s_{1} \psi_{a}\left(u_{s}\right)+s_{2} \psi_{b}\left(u_{S}\right)=\left(2 s_{1}+s_{2}\right) \frac{2^{s_{2}-1}}{3^{s_{1}+s_{2}-1}}
$$

with $a \in S_{1}$ and $b \in S_{2}$. So, we can determine $\psi_{a}\left(u_{S}\right)$ for $a \in S_{1}$. Thanks to null player we have that $\psi_{c}\left(u_{S}\right)=0$ if $c \in S_{3}$. Thus, $\psi$ coincides with the Banzhaf index for $(3,2)$-simple games for any unanimity game $u_{S}$.

We suppose that the thesis holds for any game $v$ such that $\left|\mathcal{W}^{m}(v)\right| \leq k-1$, and prove it for $v$ such that $\left|\mathcal{W}^{m}(v)\right|=k$.

If $\mathcal{W}^{m}(v)=\left\{S^{1}, \ldots, S^{k}\right\}$, then $v=u_{S^{1}} \vee u_{S^{2}} \vee \cdots \vee u_{S^{k}}$, since $\psi$ satisfies the transfer axiom:

$$
\psi(v)=\psi\left(u_{S^{1}}\right)+\psi\left(u_{s^{2}} \vee \cdots \vee u_{S^{k}}\right)-\psi\left(u_{S^{1}} \wedge u_{S^{2}} \wedge \cdots \wedge u_{S^{k}}\right)
$$




\begin{tabular}{|c|c|c|c|c|c|}
\hline & Anonymity & Null & Transfer & Total power & Yes-abstain \\
\hline$\gamma^{1}$ & - & $\checkmark$ & $\checkmark$ & $\checkmark$ & $\checkmark$ \\
$\gamma^{2}$ & $\checkmark$ & - & $\checkmark$ & $\checkmark$ & $\checkmark$ \\
$\gamma^{3}$ & $\checkmark$ & $\checkmark$ & - & $\checkmark$ & $\checkmark$ \\
$\gamma^{4}$ & $\checkmark$ & $\checkmark$ & $\checkmark$ & - & $\checkmark$ \\
$\gamma^{5}$ & $\checkmark$ & $\checkmark$ & $\checkmark$ & $\checkmark$ & - \\
\hline
\end{tabular}

Table 3: Independence of the axioms that characterize the Banzhaf index for (3,2)-simple games

The conjunction of unanimity games is still a unanimity game, so all games on the right-hand side of the previous equation have a number of minimal winning tripartitions smaller than $\left|W^{m}(v)\right|$. Using the induction hypothesis, $\psi$ coincides with the Banzhaf index for $(3,2)$-simple games for all of them and this ends the proof.

\subsection{Independence of the axiom for the Banzhaf index for $(3,2)$-simple games}

The five axioms for $(3,2)$-simple games used in Theorem 5.1 are independent. We are going to give examples of power indices on (3,2)-simple games that satisfy only four of them, as summarized in Table 3.

Not anonymity Consider the index $\gamma^{1}$ defined on unanimity games as follows.

- If $s_{3}=n-2$ then for any two players $a$ and $b$ such that $a<b$,

- if $S=(a b, \emptyset, N \backslash\{a, b\})$, then

$$
\gamma_{a}^{1}\left(u_{S}\right)=\frac{1}{3}+\varepsilon, \quad \gamma_{b}^{1}\left(u_{S}\right)=\frac{1}{3}-\varepsilon ;
$$

- if $S=(a, b, N \backslash\{a, b\})$, then

$$
\gamma_{a}^{1}\left(u_{S}\right)=\frac{2}{3}+\varepsilon, \quad \gamma_{b}^{1}\left(u_{S}\right)=\frac{1}{3}-\varepsilon ;
$$

- if $S=(b, a, N \backslash\{a, b\})$, then

$$
\gamma_{a}^{1}\left(u_{S}\right)=\frac{1}{3}+\varepsilon, \quad \gamma_{b}^{1}\left(u_{S}\right)=\frac{2}{3}-\varepsilon ;
$$

- if $S=(\emptyset, a b, N \backslash\{a, b\})$, then

$$
\gamma_{a}^{1}\left(u_{S}\right)=\frac{2}{3}+\varepsilon, \quad \gamma_{b}^{1}\left(u_{S}\right)=\frac{2}{3}-\varepsilon ;
$$

where $\varepsilon>0$. 
- If $s_{3} \neq n-2, \gamma^{1}\left(u_{S}\right)=\beta\left(u_{S}\right)$ where $\beta$ is the Banzhaf index for $(3,2)$-simple games.

Then extend $\gamma^{1}$ to $\mathfrak{T}^{N}$ using transfer.

This index satisfies null player, total power on unanimity games and yes-abstain null loss. However, $\gamma^{1}$ does not satisfy anonymity, because for instance

$$
\gamma_{a}^{1}\left(u_{(a b, \emptyset, N \backslash\{a, b\})}\right) \neq \gamma_{b}^{1}\left(u_{(a b, \emptyset, N \backslash\{a, b\})}\right) .
$$

Not null player Consider the index $\gamma^{2}$ defined on unanimity games as follows.

- If $S=(\emptyset, a, N \backslash\{a\})$ or $S=(a, \emptyset, N \backslash\{a\})$ for some $a \in N$, then

$$
\gamma_{a}^{2}\left(u_{S}\right)=1-\varepsilon \quad \gamma_{b}^{2}\left(u_{S}\right)=\frac{\varepsilon}{n-1}
$$

for any $b \neq a$ and with $\varepsilon>0$;

- for any other $S \in 3^{N}, \gamma^{2}\left(u_{S}\right)=\beta\left(u_{S}\right)$ where $\beta$ is the Banzhaf index for $(3,2)$-simple games.

Then extend $\gamma^{2}$ to $\mathfrak{T}^{N}$ using transfer.

This index satisfies anonymity, total power on unanimity games and yes-abstain null loss. However, $\gamma^{2}$ does not satisfy null player: any $b \neq a$ is a null player in the game $u_{(\emptyset, a, N \backslash\{a\})}$ but $\gamma_{b}^{2}\left(u_{(\emptyset, a, N \backslash\{a\})}\right)=\frac{\varepsilon}{n-1} \neq 0$.

Not transfer Consider the index $\gamma^{3}$ defined as $\gamma^{3}\left(u_{S}\right)=\beta\left(u_{S}\right)$ for any unanimity game $u_{S}$ and for any other game $v$

$$
\gamma_{a}^{3}(v)= \begin{cases}0 & \text { if } a \text { is a null player } \\ \frac{1}{k} & \text { otherwise }\end{cases}
$$

where $k=\mid\{p \in N: p$ is not a null player in $v\} \mid$.

The index $\gamma^{3}$ satisfies the null player and the anonymity axioms. It also satisfies the total power on unanimity games and the yes-abstain null power, since it coincides with the Banzhaf index on unanimity games. From the definition of $\gamma^{3}(v)$ it is clear that this index does not satisfy the transfer axiom.

Not total power on unanimity games Consider the index $\gamma^{4}$ defined on unanimity game as

$$
\gamma_{a}^{4}\left(u_{S}\right)= \begin{cases}\frac{1}{s_{1}+s_{2}} & \text { if } a \in S_{1} \cup S_{2} \\ 0 & \text { if } a \in S_{3}\end{cases}
$$

and extended to $\mathfrak{T}^{N}$ using transfer.

The index $\gamma^{4}$ satisfies anonymity, null player, and yes-abstain null loss. However, $\gamma^{4}$ satisfies efficiency instead of total power on unanimity game. 
Not yes-abstain null loss Consider the index $\gamma^{5}$ defined on unanimity game as

$$
\gamma_{a}^{5}\left(u_{S}\right)= \begin{cases}\frac{2 s_{1}+s_{2}}{s_{1}+s_{2}} \frac{2^{s_{2}-1}}{3^{s_{1}+s_{2}-1}} & \text { if } a \in S_{1} \cup S_{2} \\ 0 & \text { if } a \in S_{3}\end{cases}
$$

and extended $\mathfrak{T}^{N}$ using transfer.

The index $\gamma^{4}$ satisfies anonymity, null player, and total power on unanimity games. However, it does not satisfy yes-abstain null loss:

$$
\gamma_{a}^{5}\left(u_{S}\right)=\frac{2 s_{1}+s_{2}}{s_{1}+s_{2}} \frac{2^{s_{2}-1}}{3^{s_{1}+s_{2}-1}} \neq \frac{2 s_{1}+s_{2}-1}{s_{1}+s_{2}} \frac{2^{s_{2}}}{3^{s_{1}+s_{2}-1}}=\gamma_{a}^{5}\left(u_{S \downarrow a}\right) .
$$

\section{Conclusion}

In this work we provide an axiomatization for the Shapley-Shubik index for $(3,2)$-simple games. The definition of this index in Felsenthal and Machover's seminal work is given as the expected probability under the discrete uniform distribution of each player of being pivotal in the roll-call space. Thus, there was a bargaining interpretation of this index but there was not, in that original paper, an axiomatic characterization of the index and the list of the properties that it satisfies.

Our work focus on the classical axiomatization of the Shapley-Shubik and the Banzhaf indices for simple games, due to [Dubey [1975]] and [Dubey and Shapley [1979]], respectively, and generalize these approaches to the family of $(3,2)$-simple games. The characterizations we give for the two indices have a very similar structure and they can be of future interest in order to define new indices for $(3,2)$-simple games analogous to probabilistic values and semivalues defined for cooperative and simple games, as in [Dubey et al. [1981] ] and [Monderer and Samet [2002]].

Moreover, it can be of future interest to study other axiomatizations of power indices for (3,2)-simple games, not related to the behavior on unanimity games, as done in [Freixas and Lucchetti [2016]] and in [Bernardi [2017]] only for the Banzhaf index for (3,2)-simple games.

Finally, the axiom we introduce, together with anonymity and efficiency, allows to compute the Shapley-Shubik index for $(3,2)$-simple games on unanimity games using a recursive formula. Thus, using the transfer property it is possible to compute the value for any game, without explicitly use the definition and count the number of roll-calls in which a player is pivotal. Further research

could be addressed to compute the Shapley-Shubik index $(3,2)$-simple games for any $(3,2)$-simple game by means of a direct formula.

\section{Acknowledgments}

Josep Freixas acknowledges the Spanish Ministry of Economy and Competitiveness (MINECO) and the European Union (FEDER funds) under grant MTM2015-66818-P (MINECO/FEDER). 


\section{References}

[Albizuri [2001]] Albizuri, M. J.. [2001] An axiomatization of the modified Banzhaf Coleman index. International Journal of Game Theory, Vol. 33, No. 2,167-176.

[Banzhaf [1964]] Banzhaf, J.F. [1964] Weighted voting doesn't work: A mathematical analysis. Rutgers L. Rev., 19, 317-343.

[Bernardi and Lucchetti [2015]] Bernardi, G., and Lucchetti, R. [2015]. Generating Semivalues via Unanimity Games. Journal of Optimization Theory and Applications, Vol. 166, No. 3, 1051-1062.

[Bernardi [2017]] Bernardi, G. [2017] A new axiomatization of the Banzhaf index for (3,2)-simple games. Group Decision and Negotiation, 1-13.

[Carreras et al. [2003]] Carreras, F., Freixas, J., and Puente, M. A. [2003]. Semivalues as power indices. European Journal of Operational Research, Vol. 149, No. 3, 676-687.

[Dubey [1975]] Dubey, P. [1975]. On the uniqueness of the Shapley value. International Journal of Game Theory, Vol. 4, No. 3,131-139.

[Dubey and Shapley [1979]] Dubey, P. and Shapley, L.S. [1979] Mathematical properties of the Banzhaf power index. Mathematics of Operations Research, Vol. 4, No. 2,99-131.

[Dubey et al. [1981] ] Dubey, P., Neyman, A., and Weber, R. J. [1981]. Value theory without efficiency. Mathematics of Operations Research, Vol. 6, No. 1, 122-128.

[Felsenthal and Machover [1997]] Felsenthal, D.S. and Moshé Machover, M. [1997] Ternary voting games. International Journal of Game Theory, Vol. 26, No. 3,335-351.

[Felsenthal and Machover [1998]] Felsenthal, D.S. and Moshé Machover, M. [1998] The Measurement of Voting Power: Theory and Practice, Problems and Paradoxes. Edward Elgan Publishing.

[Freixas and Zwicker [2003]] Freixas, J., and Zwicker, W. S. [2003]. Weighted voting, abstention, and multiple levels of approval. Social Choice and Welfare, Vol. 21, No. 3, 399-431.

[Freixas [2005a]] Freixas, J. [2005a]. Banzhaf measures for games with several levels of approval in the input and output. Annals of Operations Research, Vol. 137, No. 1, 45-66.

[Freixas [2005b]] Freixas, J. [2005b] The Shapley-Shubik power index for games with several levels of approval in the input and output.Decision Support Systems, Vol. 39, No. 2, 185-195. 
[Freixas and Lucchetti [2016]] Freixas, J. and Lucchetti, R. [2016] Power in voting rules with abstention: an axiomatization of a two components power index. Annals of Operations Research, Vol. 244, No. 2, 455-474.

[Laurelle and Valenciano [2001]] Laruelle, A. and Valenciano, F. [2001] Shapley-Shubik and Banzhaf indices revisited. Mathematics of Operations Research, Vol. 26, No. 1,89-104.

[Lehrer [1988]] Lehrer, E. [1988] An axiomatization of the Banzhaf value. International Journal of Game Theory, Vol. 17, No. 2,89-99.

[Monderer and Samet [2002]] Monderer D. and Samet D. [2002] Variations on the Shapley value. Handbook of Game Theory with Economic Applications 3, $2055-2076$.

[Penrose [1946]] Penrose, L. S. [1946] The elementary statistics of majority voting. Journal of the Royal Statistical Society, Vol. 109, No. 1, 53-57.

[Shapley and Shubik [1954]] Shapley, L.S. and Shubik, M. [1954] A method for evaluating the distribution of power in a committee system. The American Political Science Review, Vol. 48, No. 3,787-792. 